\title{
A Whole-Arm Tactile Display System
}

\author{
Riichiro Tadakuma and Robert D. Howe \\ Harvard School of Engineering and Applied Sciences
}

\begin{abstract}
This work presents a new tactile display device for relaying contact information to locations along the human arm. The system is intended to facilitate teleoperation of whole-arm manipulation tasks, such remotely controlling a humanoid robot to grasp and lift large objects. The system consists of a set of five tactors, each a foam-covered paddle $42 \times 48 \mathrm{~mm}$. These tactors are brought into contact with the skin of the user's arm by small DC motors under computer control. The tactors are mounted on frames which are readily mounted to the upper and lower arm using Velcro straps. User tests demonstrate that the system can effectively convey contact information to the user. The benefits of this haptic display modality are evaluated in comparison with degraded visual information to estimate the resolution limits of the system.
\end{abstract}

KEYWORDS: tactile display, whole-arm manipulation, teleoperation.

\section{INTRODUCTION}

Teleoperation is, at present, the most effective method for performing robotic manipulation tasks in unstructured environments. Telemanipulation is typically performed using the robot arm's end effecter, but whole-arm manipulation can extend manipulation capabilities by using all surfaces of the arm to interact with objects in the remote environment [1-3]. This has particular promise for tasks that involve interactions between the remote robot and the human body, such as rescue operations in hazardous environments, care of the elderly and infirm, and space exploration. In general, whole-arm telemanipulation can expand the range of robotic interaction to objects that are on the same scale as the entire robot arm; for teleoperated humanoid robots with two arms, this can extend to the scale of the entire humanoid body.

A challenge in whole-arm telemanipulation is conveying the mechanical interaction in the remote environment to the operator. For example, in attempting to grasp a large object by enveloping it with the robot arm, it is important to detect the location and magnitude of the contact forces along all arm segments. This contact information helps to determine the stability of the grasp and the ability to lift the object and carry out manipulation tasks [4-5]. Sensing contact locations using vision in whole-arm manipulation is problematic due to occlusions, and the need for force information.

Previous work towards conveying contact interactions in teleoperation has largely focused on force feedback techniques for

Robert D. Howe and Riichiro Tadakuma are with the Harvard School of Engineering and Applied Sciences, 29 Oxford Street, Cambridge, MA 02138, USA (e-mail: howe@seas.harvard.edu). reproducing force vectors from remote interactions at fixed locations on the hand or fingers (e.g. [6-9]). For conveying distributed pressure or shape information across the skin, attention has focused on fingertip-scale devices (e.g. [10-11]). Across larger regions of the body, a number of systems have been demonstrated using vibrotactile stimulators [12-13] or electrotactile displays to convey spatially distributed stimuli [7], [14]. None of these systems are capable of reproducing variations in force intensity across the arm's surface.

In this paper we present a prototype lightweight, wearable tactile display system for the mechanical reproduction of contact sensation along the length of the arm. The system has modules that mount on the forearm and the upper arm. Each module consists of a set of "tactors" with a foam-covered paddle that is pressed against the teleoperator's arm by a DC servo motor under computer control. Electromagnetic trackers sense the arm's position and orientation to coordinate tactile sensation with arm motion. After describing the system design and bench-top performance, we describe evaluation of the system by relating haptic and visual resolutions.

\section{System Design}

The system is intended as a first prototype for applications involving remote control of a humanoid robot. Tactile sensors on the remote robot's arms will measure contact force locations and intensities [16-26]. The tactile display system will then reproduce this distributed force information along the operator's arm. Tactile display systems for whole-arm telemanipulation systems should be lightweight and easily mounted, and leave the hand free for other control functions.

The system is intended to provide sufficient resolution, in both force magnitude and spatial distribution, to enable a variety of whole-arm telemanipulation tasks. At present, the tactile fidelity requirements to enable this kind of task performance are not known; indeed, one of the motivations for the construction of this first-of-its-kind tactile display system is to explore these performance requirements. Initial design specifications must thus be inferred from the neurophysiology and psychophysics of the human tactile sensing system, as well as prospective task requirements. The hairy skin of the human arm has a two-point discrimination and gap detection thresholds on the order of $40 \mathrm{~mm}$ [27]; this is greatly reduced resolution compared to the hand,

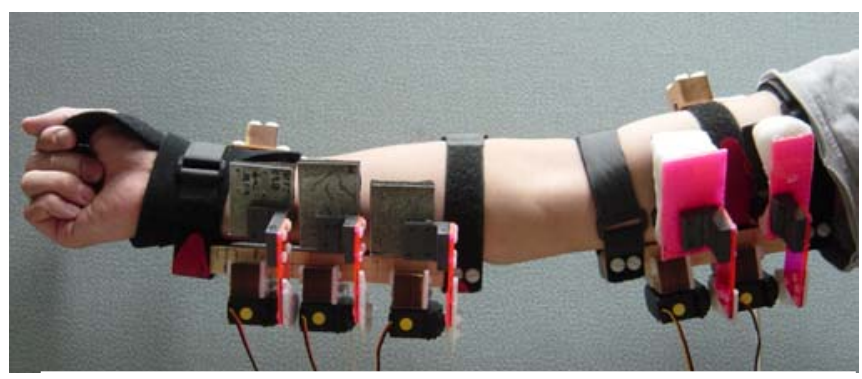

Figure 1. Tactile display mounted on user's arm. 


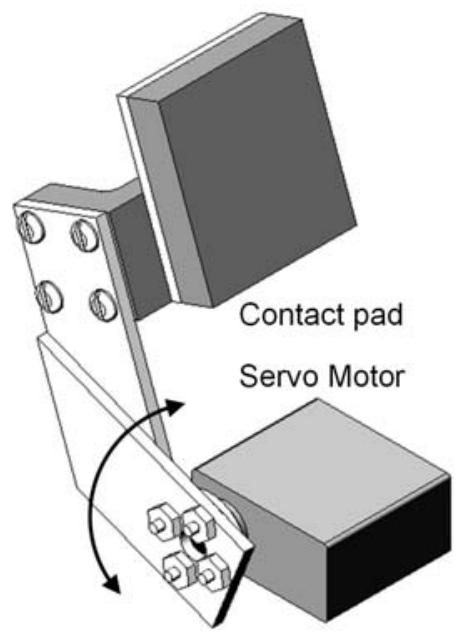

Figure 2. A tactile display actuator.

where two-point thresholds are a few $\mathrm{mm}$ on the fingertips. Perceptual thresholds for force are similarly reduced. The force/indentation threshold on the arm is $142 \mathrm{mg}$, while on the fingertip is $76 \mathrm{mg}$ at low frequencies. While such measures do not directly map to tactile display performance specifications [28], they suggest that a small number of display units on the arm can convey the needed information.

In light of this low resolution requirement, our system uses five tactile actuators or "tactors" (Fig. 1), with three evenly spaced along the volar forearm and two on the upper arm; the asymmetry is due to the anticipated greater role of the forearm in establishing contact and maintaining stability in whole-arm grasping. Each tactor unit is actuated by a small, high performance servo motor (Fig. 2) developed for use in radio-controlled (RC) hobby applications (CS-60, HOBBICO, Champaign, IL, USA). These servo motors have a manufacturer's specified maximum output torque of $3.06 \mathrm{~kg}-\mathrm{cm}$ and a maximum speed of $0.19 \mathrm{~s} / 60 \mathrm{deg}$. This results in an inexpensive prototype system with reasonably good performance for initial evaluation of the benefits of this type of feedback.

The output shaft of each tactor unit is connected to an acrylic link ending in a paddle that presses against the arm. Each link is shaped so that at contact the paddle is moving approximately orthogonal to the skin. The dimensions of the paddle are $42 \mathrm{x}$ $48 \mathrm{~mm}$. The paddles are covered with a $10 \mathrm{~mm}$ layer of complaint foam rubber with Young's modulus of $8 \mathrm{kPa}$ to minimize stress concentrations.

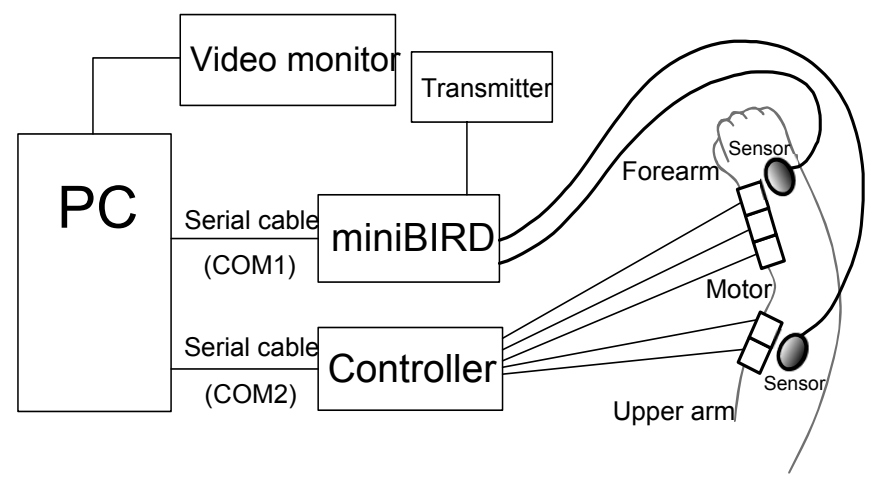

Figure 3. Block diagram of experimental setup.
The display frame is attached to the user's arm by neoprene straps secured by Velcro strips. The total mass of the display for forearm is $362 \mathrm{~g}$, and its dimensions are $73 \mathrm{~mm} \mathrm{x} 115 \mathrm{~mm} \mathrm{x}$ $215 \mathrm{~mm}$. The upper arm display mass is $353 \mathrm{~g}$, and its dimensions are $71 \mathrm{~mm} \times 145 \mathrm{~mm}$ x $160 \mathrm{~mm}$.

The five servo motors are controlled by a PWM interface chip (FT639, E-LAB Digital Engineering, Inc., Independence, MO, USA) through an RS-232C port. The servo motor modules include a position controller, with an effective displacement resolution at the paddle of $0.49 \mathrm{~mm}$ per bit.

The motion of human arm is measured with an electromagnetic motion tracking system (miniBIRD 800, Ascension Technology Inc.). Two sensors are used in this system. One is on the forearm near the wrist, and the other is on the upper arm (Fig. 3). To prevent undesirable electromagnetic interference between the tactile display system and the electromagnetic sensors, the display frame is constructed from nonmetallic materials. The sensor is mounted on the top of the subject's arm, to maximize the distance from the motors. Careful testing revealed that at this distance there was no measureable effect on the tracker from activation of the motor.

The display system is easily mounted on the user's arm, and the straps permit adaptation to a wide variation in arm sizes. Following mounting, the offset position of each tactor paddle is adjusted using a software utility to accurately align the initial contact position for each tactor. During operation, the tactor position is maintained just out of contact with the skin until display of contact is commanded.

\section{Evaluation}

A complete evaluation of the pertinent benefits and performance characteristics of this type of tactile display system is challenging. Initial tests readily confirm that the system is successful in conveying contact sensations. To provide a more sophisticated appraisal, we developed an experimental paradigm that compared task performance with the tactile display system to execution of the same task with only visual feedback. This provides an immediate measure of the added capabilities provided by adding tactile feedback to teleoperation of a humanoid robot.

\subsection{Experimental setup}

Subjects were asked to complete a task that consisted of grasping a cylinder using both the upper and lower arm segments (Fig. 4). The task is performed in a planar virtual environment, which simplifies implementation and enables collection of complete task-related data, and removes the complication of distinguishing the display system performance from the performance limitations

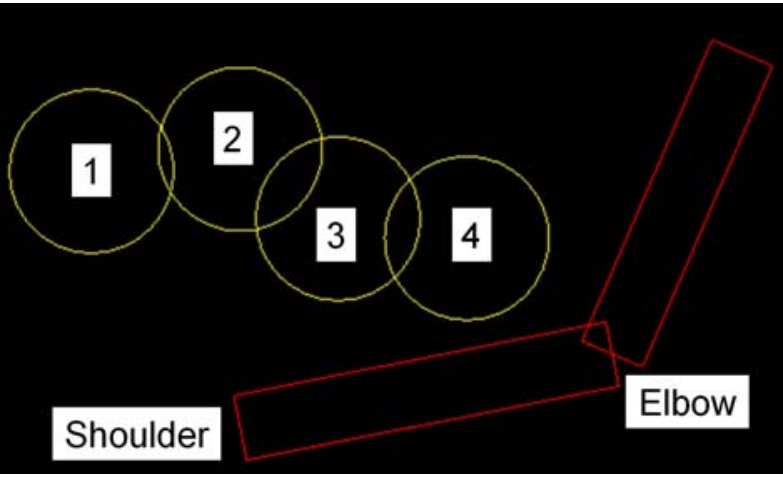

Figure 4. Relative positions of the virtual objects and the slave robot arm 


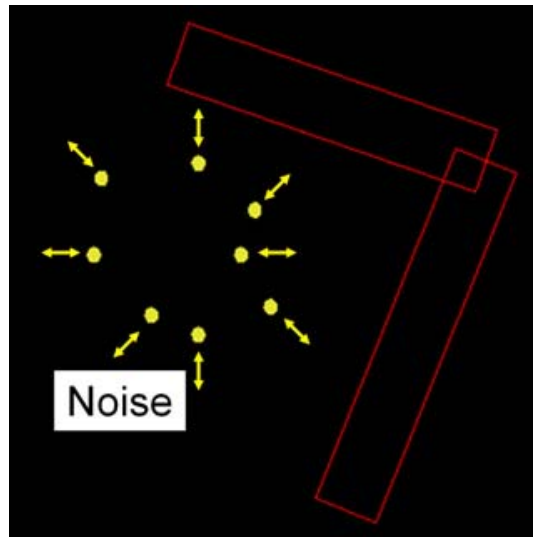

Figure 5. The target object is represented as dots whose positions are jittered ("visual noise") to quantitatively reduce visual information.

of a physical humanoid robot. Subjects viewed a visual display on a computer monitor of a two-segment wireframe representation of a "remote robot arm," i.e. a virtual slave robot. The upper and lower segments of this arm are controlled by orientation readings from the electromagnetic tracker sensors on the tactile display system. Because the virtual arm joints are controlled by sensed orientation of the user's arm, the relative size of the object compared to the user's arm length is the same for all subjects.

At the start of each trial, subjects are presented with a disk representing the object to be grasped. The initial location of the object is varied among four positions for each trial to minimize learning of successful arm trajectories. Subjects are instructed to use their own arm to control the virtual arm to grasp the object by making simultaneous contact with both the upper and lower arm segments. The virtual simulation detects contact between the arm and the object, which generate virtual forces against the object in proportion to the small interpenetration of the arm and object surfaces. The object can slide with friction over the planar workspace in response to these forces, and the object must be pushed to an appropriate location for grasping. The virtual arm and object simulation was implemented in OpenGL and Visual $\mathrm{C}++6.0$.

The tactile display system is activated in response to these virtual contacts. The forward edge of each arm segment is divided into regions corresponding to each display tactor. Each tactor is activated in proportion to the virtual force generated by contact and interpenetration with the object within its region on the virtual arm surface. Using the system subjects can readily detect initial contact and distinguish the contact intensity.

To provide a quantitative comparison of the utility of tactile feedback versus visual feedback, for some trials we reduced visual information about the object location displayed on the monitor. This was accomplished by displaying the object not as a complete circular outline, but as a set of dots equally spaced along the object perimeter. These dots were then actively perturbed or "jittered" randomly in the radial direction to obscure the exact position of the object (Fig. 5). New dot locations were displayed 6 times per second at Gaussian distributed locations, with variable standard deviation and a mean location exactly on the object boundary.

\subsection{Experimental protocol}

Subjects $(n=12)$ were undergraduate university students who voluntarily participated following a protocol approved by the Harvard University human subjects committee. Subjects were given time to practice the task using the system, initially with the

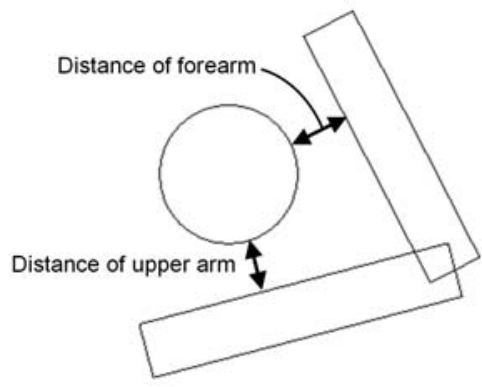

Figure 6. Definition of the distance from the arm to the object

complete outline of the object, then with the fixed dot representation, then with various noise levels. Subjects continued until they successfully grasped the object at least two times under each of these conditions.

Following the practice phase, experimental trials used four values for the visual noise standard deviations $(0.1,0.333,1.0$, and 3.33 units) and four initial positions for the object (Fig. 4). In addition, in alternate trials the tactile display was turned off, to compare visual+haptic vs. visual-only conditions. Each subject completed a total of 64 trials in a balanced Latin square presentation. Auditory cues were masked with headphones playing prerecorded sounds of the servo motors.

Subjects were instructed to grasp the object precisely and gently. Performance was evaluated in terms of distance from the edge of the robot arm to the edge of the object when the subject declared orally that they had grasped the object (Fig. 6). Note that distances could assume both positive values (i.e. no contact) or negative values (interpenetration).

\subsection{Results}

Fig. 7 shows the mean distance between the object and the arm at the end of the trial, calculated as the sum of the upper and lower arm distances, for all trials by all subjects. For the visual+haptic feedback case, increasing levels of visual noise have little effect on performance. With the tactile display active, subjects on average produced a slight interpenetration (negative distance) between the object and arm for all values of visual noise. The scaling between the virtual distance units (as plotted in Fig. 7) and the physical distance at the subject's arm is $129 \mathrm{~mm} / \mathrm{unit}$, so interpenetration values ranged from 8.8 to $13.8 \mathrm{~mm}$ with a mean of $10.3 \mathrm{~mm}$ across all values of visual noise.

In contrast, mean distances for the visual-only case become increasingly positive with increasing visual noise. Positive values indicate that the arm is not in contact with object, and thus the object is not successfully grasped. Distances at the subject's arm increase from about $-5 \mathrm{~mm}$ at the lowest visual noise up to about $25 \mathrm{~mm}$ for the highest visual noise levels. Paired $t$-tests show that means for visual+haptic and visual-only are significantly different for all four noise levels $(p<0.001)$. (Note that the large number of samples -12 subjects $\times 8$ trials for each condition - permit statistical differentiation despite the large standard deviations shown in Fig. 8.)

Fig. 8 shows the standard deviation of the object-arm distance as a function of visual noise level across all subjects. As with the mean distances, for the visual+haptic case the standard deviation of distance is essentially constant at approximately $10 \mathrm{~mm}$ irrespective of the visual noise level. For the visual-only feedback case, the standard deviation of the distances increases markedly for the highest visual noise levels (129 and $430 \mathrm{~mm}$ ). These noise 


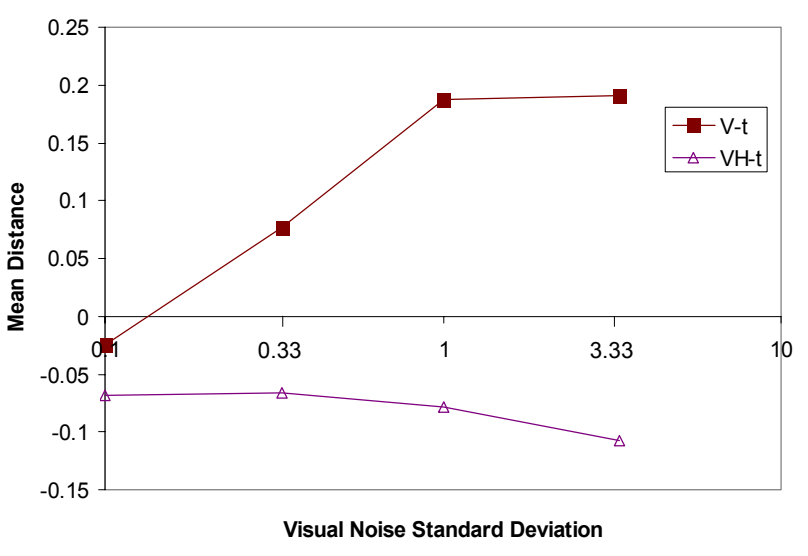

Figure 7. Mean distance between the object and arm (sum of upper and lower arm distances) as a function of visual noise standard deviation. VH-t: Visual and haptic information; V-t: Visual only.

Standard Deviations of Distances

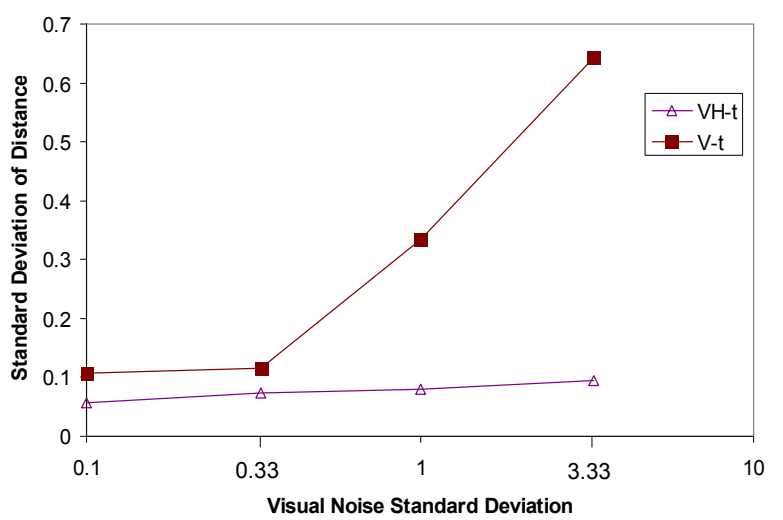

Figure 8. Standard deviation of distance between the object and arm (sum of upper and lower arm distances) as a function of visual noise standard deviation.

levels made the task quite difficult; for the visual-only condition, subjects often reported that they were essentially guessing the object location when only the highest-noise visual information was available.

\section{Discussion}

These results confirm that a simple whole-arm tactile display system can convey useful information for telemanipulation tasks. With tactile feedback subjects were able to consistently grasp the virtual object, even when visual information was essentially absent. This is a key capability in whole-arm telemanipulation tasks, where the robot arm and the object will often occlude the intended contact locations. Because the goal is to attain firm contact, even the simple tactile display system presented here can convey crucial information.

\subsection{Packaging and costs}

The prototype system is lightweight, easily mounted, and comfortable to wear. Frame and strap construction are simple and inexpensive. Costs for the tactor system are also low, as it is based on consumer servo motors that include a DC motor, power amplifier, position controller, and PWM signal decoder in a small, robust and inexpensive package. These devices are intrinsically position controlled, which may be appropriate for interaction with rigid objects. To relay contact information from manipulation of complaint objects, force control of the tactors may be appropriate.

The prototype system cost is dominated by electromagnetic tracking system for measuring the human operator's arm position. Less expensive alternatives might include video-based tracking, or a mechanical goniometer system to directly measure arm joint angles that could be integrated with the tactor frame. We note that any teleoperated system will need to measure arm positions to provide motion commands to the remote robot arm, so in this sense operator arm tracking is an existing capability in such systems.

\subsection{Performance evaluation}

One of the goals of this project is to begin determining the performance requirements for whole-arm tactile display systems. This is a complex issue, as whole-arm telemanipulation requires arm segment positioning and force application in both normal and lateral directions. These parameters must be modulated in real time in response to sensed object properties. The creation of effective and efficient tactile display systems will require the determination of the role of haptic guidance in enhancing performance of these functions.

In this initial study we focused on a simple grasping task that examines the positioning performance aspects. The results show that operators can use the tactile display to control position relative to the object with good accuracy and repeatability (Figs. 7 and 8). Subjects regularly produced a slight interpenetration, with a mean object-arm distance of $-10.3 \mathrm{~mm}$. This represents relatively precise motor control, as subjects were required to hold their arm at a particular configuration in space with no external forces or mechanical constraints. Consistency in positing relative to the object was also high, with a mean standard deviation of distance of $9.8 \mathrm{~mm}$. These results are nearly constant across all levels of visual noise, including the highest visual noise level where visual information is essentially absent.

The observed interpenetration that subjects produced with the tactile display active is needed to activate the tactile display, and these values are consistent with subjects relying on the haptic feedback to discern the correct relationship between the arm and object for grasping. The visual-only condition produced mean interpenetration only at the lowest noise level. At higher noise levels with only visual feedback, subjects appear to consistently overestimate the location of the object edge in the radial direction, so the mean distance is positive, resulting in noncontact. This may be specific to the visual noise display method used here, where visual noise is implemented as radial motion of the dots denoting the object edge. It does, however, demonstrate how whole-arm manipulation abilities will be limited with decreasing visual information.

\subsection{Relating haptic and visual variability}

This data also permits quantitative comparison of haptic to visual perception in terms of the effective accuracy and repeatability in this task. Basing this comparison on the mean distance measurements (Fig. 7) is problematic, because subjects apparently overestimate the object edge position when only noisy visual information is provided, as noted above. The standard deviation of distance (Fig. 8), however, permits estimates of relative trial-totrial variability, in effect estimating the relative noise level on each sensory channel. 
These results show that the visual+haptic standard deviation of object-to-arm distance is approximately equal to visual-only standard deviation for the two low visual noise cases. For higher visual noise levels, visual-only becomes far more variable while visual+haptic remains almost unchanged. This suggests that the haptic and visual perceptual variability are approximately equal near the point where the curves diverge, i.e. visual noise with standard deviation of 0.33 units or $43 \mathrm{~mm}$. This value thus serves as an estimate of the equivalent noise for the haptic perceptual channel.

This is consistent with the results of Ernst and Banks [15], who show that the central nervous system combines visual and haptic sensory information in a manner that approximates maximum likelihood estimation. This means that input from each sensory channel is evaluated according to its variability, so that lower noise channels are accorded more weight in estimating perceptual quantities.

In the present experiment, haptic sensory variability is presumably constant, while the visual channel's variability was systematically controlled through the addition of visual noise. The curves in Fig. 8 follows the expected form for statistically optimum estimation under these conditions [15]. A full analysis of these results using the maximum likelihood framework is beyond the scope of this paper, but could lend further insight into the relationship between visual and tactile feedback in this task setting, and contribute to the understanding of the resolution requirements for whole-arm tactile displays. This approach of using the maximum likelihood estimation framework as a means of determining the relative information content of different sensory channels may be a useful means of evaluating multimodal interfaces in many settings.

\subsection{Future work}

While the system presented here proved successful in conveying essential contact information, testing was limited to virtual task execution in two dimensions. Experiments with physical slave robots and three-dimensional tasks will be essential to establish the needed parameters of tactile displays for whole-arm telemanipulation. Among the questions to be answered are the optimum number and density of tactors, required bandwidth, and the role of force vs. position control of the display. It is clear that the onset, location, and intensity of contacts are key parameters for the display to convey, but the importance of other phenomena, such as slip and local surface curvature or features (e.g. edges and corners) must be determined.

System-level design must also be addressed. Most important is the integration of the tactile display with tactile sensors on the remote robot's arms. Large-area sensors are under development and some have been commercialized [16-22], and many humanoid robots have been developed which incorporate tactile skin sensors [23-26]. How to best transform the sensor signals into appropriate drive signals for the display will require analysis of the sensor mechanical properties and signal encoding, as well as better understanding of the perceptual and motor control issues [29].

\section{Conclusions}

This paper presented the design and initial evaluation of a new type of tactile display system, intended for relaying contact information in teleoperation of whole-arm manipulation tasks. The system is lightweight and easily mounted on the operator's arm. Costs for the display system components are low due to the use of consumer RC servomotors as actuators.

Experimental validation showed that the system can convey information about contact location and intensity that allowed subjects to grasp large objects in a virtual environment. By varying the noise level in the visual information on object location, it was possible to estimate the relative information conveyed by the haptic and visual channels. Results show that the tactile display system allows accurate arm control even in the absence of visual information.

\section{ACKNOWLEDGMENTS}

The authors wish to thank the members of Harvard Biorobotics Laboratory for assistance in developing the experimental protocol and for comments on drafts of this paper. This work was supported by a grant from the Japan Society for the Promotion of Science and by the Harvard School of Engineering and Applied Sciences.

\section{REFERENCES}

[1] Salisbury, K. Townsend, W. Ebrman, B. DiPietro, D. Preliminary design of a whole-arm manipulation system (WAMS), Proceedings IEEE International Conference on Robotics and Automation, April 24-29 1988, Philadelphia, PA, USA, vol. 1, pp. 254-260.

[2] Song, P. Yashima, M. Kumar, V. Dynamic simulation for grasping and whole arm manipulation. Proceedings IEEE International Conference on Robotics and Automation, April 24-28, 2000, San Francisco, CA, USA, vol. 2, pp. 1082-1087.

[3] Asano, F. Zhi-Wei Luo Yamakita, M. Hosoe, S. Dynamic modeling and control for whole body manipulation Proceedings. 2003 IEEE/RSJ International Conference on Intelligent Robots and Systems, 2003. (IROS 2003). 27-31 Oct. 2003, vol. 3, pp. 31623167

[4] Bicchi, A.: On the problem of decomposing grasp and manipulation forces in multiple whole-limb manipulation. Journal of Robotics and Autonomous Systems, vol. 13, pp. 127-147, 1994.

[5] J.C. Trinkle, R.C. Ram, A.O. Farahat, and P.F. Stiller. Dexterous manipulation planning and execution of an enveloped slippery workpiece. In Proceedings IEEE International Conference on Robotics and Automation, volume 2, pages 442-448, May 1993.

[6] http://www.sensable.com/

[7] Kaczmarek, K.A. Haase, S.J. Pattern identification and perceived stimulus quality as a function of stimulation waveform on a fingertip-scanned electrotactile display. : IEEE Transactions on Neural Systems and Rehabilitation Engineering, 11(1): 9-16, March 2003.

[8] Bouzit M., Burdea G., Popescu G., and Boian R. The Rutgers Master II-New Design Force-Feedback Glove, IEEE/ASME Transactions on Mechatronics, 7(2), JUNE 2002

[9] http://home.novint.com/

[10] Wagner C.R., Lederman S.J., and Howe R.D., "A tactile shape display using RC servomotors," Proceedings 10th Symposium on Haptic Interfaces for Virtual Environment and Teleoperator Systems. HAPTICS 2002, Orlando, FL, USA, pp. 354-355, 2002.

[11] Wang, Q. and Hayward V. Compact, Portable, Modular, Highperformance, Distributed Tactile Transducer Device Based on Lateral Skin Deformation. Proc. 14th Symposium on Haptic Interfaces For Virtual Environment And Teleoperator Systems, IEEE, 2006, pp. 67-72.

[12] Jones L.A., Nakamura M., and Lockyer B., "Development of a Tactile Vest," Proceedings of the 12th International Symposium on Haptic Interfaces for Virtual Environment and Teleoperator Systems, pp. 82-89, 27-28 March 2004.

[13] Piateski E. and Jones L., "Vibrotactile Pattern Recognition on the Arm and Torso," Eurohaptics Conference, 2005 and Symposium on 
Haptic Interfaces for Virtual Environment and Teleoperator Systems, 2005. World Haptics 2005, First Joint, pp. 90-95, 18-20 March 2005.

[14] H. Kajimoto, Y. Kanno, S. Tachi, "Forehead Electro-tactile Display for Vision Substitution," Proceedings of EUROHAPTICS 2006, pp. 75-79, Paris, France, July 2006.

[15] Marc O. Ernst, Martin S. Banks, "Humans Integrate visual and haptic information in a statistically optimal fashion," NATURE VOL 415, 24 JANUARY 2002.

[16] http://www.nitta.co.jp/product/mechasen/sensor/kinotex_top.html

[17] Cheung, E. Lumelsky, V., "Development of sensitive skin for a 3D robot arm operating in an uncertain environment," 1989 IEEE International Conference on Robotics and Automation, pp. 10561061, Scottsdale, AZ, USA, 14-19, May, 1989.

[18] Iwata, H. Sugano, S., "Whole-body covering tactile interface for human robot coordination," 2002 IEEE International Conference on Robotics and Automation (ICRA2002) pp. 3818-3824, Washington, D.C., USA, 11-15 May, 2002.

[19] T. Mukai, M. Onishi, S. Hirano and Z.W. Luo, "Development of Soft Areal Tactile Sensors for Human-Interactive Robots," Proceedings of the 5th IEEE Conference on Sensors, B3L-D-5, Oct. 2006.

[20] Y. Ohmura, A. Nagakubo, Y. Kuniyoshi, "Conformable and Scalable Tactile Sensor Skin for Curved Surface," 2006 IEEE International Conference on Robotics and Automation, pp. 13481353, Orlando, Florida, USA, May 15-19, 2006.

[21] T. Hoshi, A. Okada, Y. Makino, and H. Shinoda, "A Whole Body Artificial Skin Based on Cell-Bridge Networking System," Proc. 3rd International Conference on Networked Sensing Systems (INSS 2006), pp.55-60, 2006.

[22] H. Chigusa, Y. Makino, H. Shinoda, "Large Area Sensor Skin based on Two-Dimensional Signal Tranmission Technology," Proceedings of World Haptics 2007, pp. 151-156, Tsukuba, Japan, 22-24 March, 2007.

[23] http://www.bmc.riken.jp/ RI-MAN/index_us.html

[24] W. Bluethmann, R. Ambrose, M. Diftler, S. Askew, E. Huber, M. Goza, F. Rehnmark, C. Lovchik, and D. Magruder, "Robonaut, a Robot Designed to Work with Humans in Space," Autonomous Robots, vol. 14, pp. 179-197, 2003.

[25] T. Odashima, M. Onishi et al., "A Soft Human-Interactive Robot RIMAN," Video Proceedings of the IEEE/RSJ International Conference on Intelligent Robots and Systems (IROS), Oct. 2006.

[26] Y. Ohmura, A. Nagakubo, and Y. Kuniyoshi, "Development of humanoid robot with whole body distributed tactile sensor," Robotics and Mechatronics Conference 2007, Japan, 2007.

[27] Weinstein S, "Intensive and extensive aspects of tactile sensitivity as a function of body part, sex and laterality," in Kenshalo DR (ed): The Skin Senses, pp. 195-222, Thomas, Springfield IL, 1968.

[28] J.C. Craig, Johnson K.O. (2000) The Two-Point Threshold: Not a Measure of Tactile Spatial Resolution. Current Directions in Psychological Science 9 (1), 29-32.

[29] Feller, RL, Lau, CKL, Wagner, CR, Perrin, DP, Howe, RD. "The Effect of Force Feedback on Remote Palpation," 2004 IEEE International Conference on Robotics and Automation, New Orleans, 2004 